\title{
Compound motor action potentials and mechanical failure during sustained contractions by electrical stimulation in paraplegic patients
}

\author{
E Rabischong ${ }^{1}$, P-L Doutrelot ${ }^{2}$ and F Ohanna ${ }^{1}$ \\ ${ }^{1}$ Centre Propara, Laboratory of Clinical Neurophysiology, Parc Euromedecine, 34195 Montpellier cedex 5; \\ ${ }^{2}$ Hôpital Nord, Service de Rééducation Fonctionnelle, 80054 Amiens cedex 1, France
}

In nine paraplegic patients we recorded the torque output and compound motor action potentials (CMAPs) produced by the quadriceps muscle during an isometric contraction elicited by electrical stimulation. The torque, the peak to peak amplitude, the latency, the peak to peak duration and the total surface of the rectified CMAPs were computed over a period of $126 \mathrm{~s}$. After a brief increase the mechanical output rapidly decreased and reached a stable minimum level by the end of $126 \mathrm{~s}$. The final torque output values ranged from 7.1 to $54 \%$ of initial values. This torque decrease was related neither to length of time between injury and testing, nor to the thoracic level of the spinal cord injury. The peak to peak amplitude of the CMAPs changed over the course of stimulation. It was noted to increase over a period of time after which it decreased to a minimum level. The latency from the onset of stimulation to the onset of the CMAP varied to a relatively small extent compared to the peak to peak duration. Therefore, the conduction velocity along the muscle fibres appeared to be more affected by the test than by the conduction velocity along the nerve fibres and the transmission across the neuromuscular junction. The mechanisms involved in the changes in CMAPs and the change in torque output over the time course of stimulation are discussed.

Keywords: fatigue; electrical stimulation; surface action potential; quadriceps; torque; paraplegic subjects

\section{Introduction}

The mechanisms of muscle fatigue remain incompletely understood though some progress has been made in recent years. Muscle fatigue is considered to be an inability to maintain force output during sustained or repetitive contractions. ${ }^{1}$ The rate and amount of muscle fatigue varies among subjects. ${ }^{2,3}$ Both the physical and myoelectric manifestations of muscle fatigue can be measured as a function of time.

Recently, a number of studies have used the compound motor action potential (CMAP) and the motorunit action potential (MUAP) to characterise muscle fatigue.$^{4,5}$ Changes in the amplitude and the area of the potentials have been measured as indices of neuromuscular-propagation failure. In different investigations a number of muscles have been studied: gastrocnemius, ${ }^{2}$ adductor pollicis, ${ }^{5,6,8}$ tibialis anterior. ${ }^{8}$ While intermittent tests are generally conducted to measure fatigue ${ }^{4-7}$ continuous tests appear appropriate to characterise muscle fatigue of the quadriceps muscle during standing. In fact, the quadriceps muscle supplies the main force for standing up and remaining standing using electrical stimulation in paraplegic people.

The electrical changes encountered during fatigue have not been found to be related to the mechanical changes. ${ }^{2,4,5}$ Therefore, we chose to characterise the electromechanical behaviour of the quadriceps muscle in the face of sustained electrical stimulation and to relate the myoelectric manifestations of muscle fatigue to the decrease in torque production.

Most of the results reported in the literature have focused on voluntary contractions. In the present study, the quadriceps muscle electrically stimulated represents an interesting isolated functional contractile tissue independent of motivational factors. Since the quadriceps was deprived of voluntary control only the peripheral mechanisms of fatigue will be discussed.

\section{Methods}

\section{Population}

Tests were performed on nine paraplegic patients. All had a complete spinal cord lesion at the thoracic level (ranging from T6 to T10) verified by clinical examination. None had spasticity or intermittent spasms which would have impeded the tests. The quadriceps reflex was present in each patient. The length of time between injury and testing ranged from 8 to 60 months (average $30 \pm 19.1$ ). The mean age of the subjects was 29 years $( \pm 4.1)$. All subjects gave informed consent to participate in this investigation, and this study was approved by the local ethical committee. 


\section{Measurements}

We measured both the torque output and the compound motor action potentials (CMAPs) of an electrically stimulated quadriceps muscle. The subjects sat in a special chair with hips flexed to $90^{\circ}$ and knees to $60^{\circ}$, which corresponds to the position where the maximum isometric torque has been recorded in other studies of paraplegic people using electrical stimulation., 9,10 To record the torque data, we used a force transducer (bridge of strain gauges) which has been previously described.$^{10}$ The force transducer was padded to avoid polysynaptic withdrawal reflexes due to nociceptive segmented reflexes. The bridge voltage supplied was $\pm 5 \mathrm{~V}$. The signal was amplified (Dynagraph R.611, Sensormedics Co, Anaheim, CA, USA) and low-pass filtered $(30 \mathrm{~Hz})$.

The muscle was supramaximally stimulated for $126 \mathrm{~s}$ through a pair of Nicolet electrodes (Nicolet Instruments, Trappes $\mathrm{Cx}$, France $0.9 \mathrm{~cm}$ diameter, $3 \mathrm{~cm}$ between electrodes) placed on the skin over the femoral nerve, just medial to the sartorius, at groin level. The cathode was proximal. The electrodes were connected to a current-regulated electrical stimulator which delivered biphasic charge-balanced pulses (150 mA max) sufficient to produce supramaximal stimulation. The level of stimulation was individually adjusted using a digital oscilloscope to monitor the CMAPs. Stimulation was considered supramaximal when CMAPs amplitude no longer increased despite an increase in the level of stimulation. The frequency was set at $20 \mathrm{~Hz}$ with a pulse width of $300 \mu \mathrm{s}$.

For the CMAP recording we used surface electrodes (Beckman Instruments, Gagny, France, $0.8 \mathrm{~cm}$ internal diameter) placed on the skin over the vastus medialis, where the best response was obtained, $(10 \mathrm{~cm}$ above the upper part of the patella) and separated by $1 \mathrm{~cm}$. The ground electrode was placed on the skin between the stimulation and the recording electrodes. The CMAPs were amplified with a differential amplifier (Tektronix AM 502, Tektronix, Les Ulis Cx, France) and filtered (bandpass $10 \mathrm{~Hz}$ to $1 \mathrm{kHz}$ ).

Signals coming from the force transducer and from the surface electrode were acquired using an analog to digital converter (12 bits resolution). Data were stored in a personal computer. Data were sampled at $15 \mathrm{kHz}$ and the acquisition was triggered every $2 \mathrm{~s}$ by the stimulation pulses. This very high sampling frequency was necessary to obtain accuracy in the time domain (resolution $0.066 \mathrm{~ms}$ ) and, therefore, to have a precise measurement of the latencies and duration. The time window for the acquisition was set at $100 \mathrm{~ms}$, ie two consecutive CMAPs.

\section{Data analysis}

Concerning the torque output, a mean value of the signal obtained through the force transducer during the $100 \mathrm{~ms}$ acquisition period was computed. We studied both the amount of decrease and the rate of decrease. For the amount of decrease, we computed the maximum torque (absolute and relative), the time to reach this maximum, the minimum relative torque, the total decrease from maximum to minimum value, the decrease from the initial value, the duration of torque decrease and the residual torque during the last measurement. For the rate of decrease, ie the speed at which the torque decreased, we computed three different slopes

$$
\text { Slope }=\frac{\Delta T}{\Delta t},
$$

where $T$ is the torque and $t$ the time, and Slope expressed in Torque $\mathrm{s}^{-1}$ at the initiation of torque production (computed between data at $t=0$ and data at $t=2 \mathrm{~s}$ ), during the main decrease (maximum slope value) and at the end of the test (mean slope value computed between the last four data recorded). A positive value means a torque increase while a negative value means a torque decrease. Considering the CMAPs (Figure 1), we computed the peak to peak amplitude (PTP) as:

$$
\mathrm{PTP}=\operatorname{Max}_{\mathrm{CMAP}}+\left|\operatorname{Min}_{\mathrm{CMAP}}\right|
$$

where Max $_{\text {CMAP }}$ is the maximum value of the considered CMAP, Min ${ }_{\text {CMAP }}$ is the minimum (absolute value) and PTP is expressed in $\mathrm{mV}$.

To discriminate the conduction velocity along the nerve fibres and along the muscle fibres we studied three different latencies: L0 from the onset of stimulation to the onset of the CMAP where the nerve conduction velocity (NCV) and the neuromuscular junction transmission (NMJ) were studied, L1 from the onset of the CMAP to the maximum amplitude of the first peak of the CMAP and L2, the peak to peak duration, from the maximum to the minimum of the CMAP were the MCV was studied.

We also computed the total rectified surface $(\mathrm{S})$ of the CMAPs as:

$$
S=P \sum_{0}^{n}|A(t)|
$$

where $P$ is the sampling period, $A$ the amplitude of the CMAP and $t$ the time; $n$ determines the CMAP duration. To eliminate negative values, the CMAP was

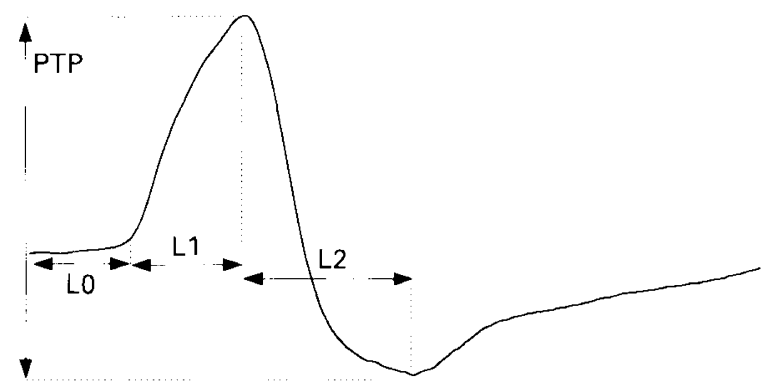

Figure 1 Measurements computed for CMAPs. PTP is noted for the peak to peak amplitude, L0 for the latency from the onset of stimulation to the first peak, L1 for the duration from the onset of CMAP to its maximum and L2 for the duration from the first peak to the second 
rectified (absolute values). $S$ (voltage-time integral) is expressed in $\mathrm{mV} \times \mathrm{ms}$.

We relate the torque output to these data.

\section{Results}

\section{Torque output}

The recorded fatigue curves revealed the non-linearity of muscle output as a function of time (sigmoid shape). As a general rule, the initial output rapidly increased (potentiation) during the first seconds of the test, reaching a maximum after which the output decreased with a slope which varied among patients (Figure 2, Table 1). However, this increase was limited (less than $15 \%$ ) in eight patients out of nine. The time to reach the maximum was usually mainly less than $6 \mathrm{~s}$ (range 2-16s). A large variability was observed among patients with respect to the maximum absolute torque produced (from 38.85 to $71.8 \mathrm{Nm}$ ) and to the residual torque output (from 7.13 to $53.54 \%$ of initial output). Neither the level of injury nor the length of time after injury correlated with residual output or maximum torque. However, patient 9 who demonstrated the best performance in terms of relative residual output $(53.54 \%)$ had the shortest time between injury and testing ( 8 months). Patient 4 , injured for the longest period of time (60 months) had the most powerful quadriceps $(71.8 \mathrm{Nm})$. We did not find any relation between the maximum absolute torque and the residual
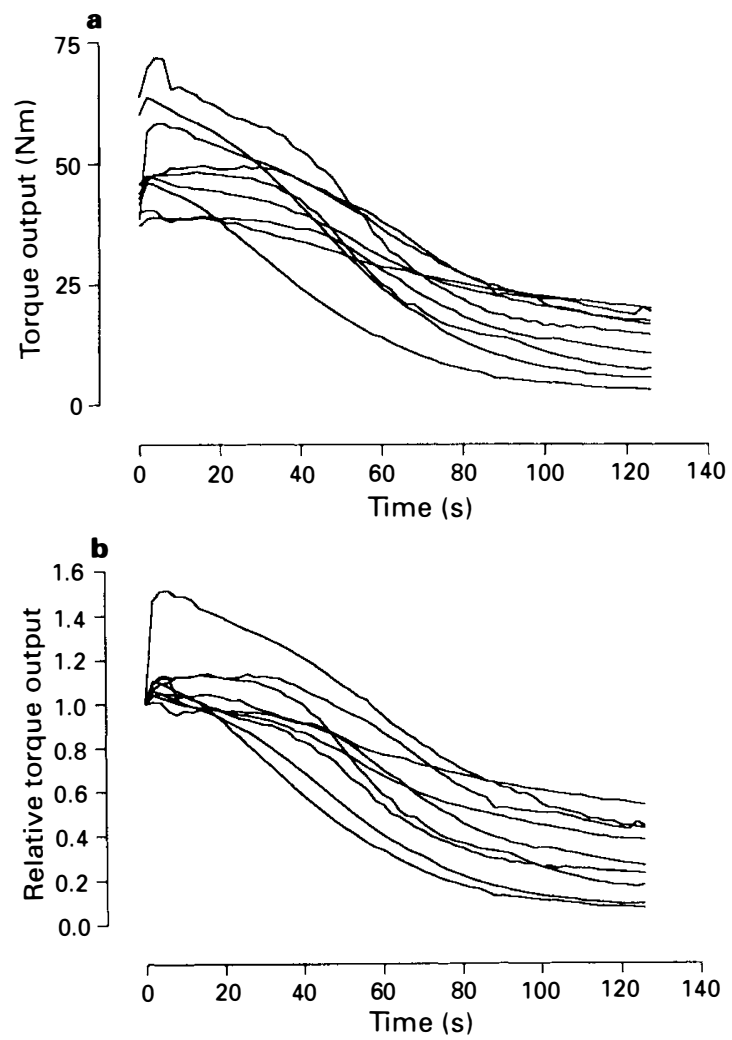

Figure 2 (a) Absolute value of torque recorded in the patients and expressed in $\mathrm{Nm}$; (b) values are normalised with respect to initial value output by the end of the test. The minimum relative torque value was equal to the residual torque value in seven out of the nine patients, and was less than $6 \%$ lower in the other patients. Therefore, no compensation for fatigue, eg recruitment of other motor units or recuperation of the stimulated motor units, was noted.

Concerning the rate of decrease, there also appeared to be a great variability among the patients. The positive value of the initial slope confirmed the initial torque increase. Except in patient 8, the initial slope was of low value and accounted for the limited torque increase. Due to the typical sigmoid shape of the curves, the maximum slope decrease was encountered at the inflexion point. This maximal value, except in patient 6 , was computed roughly at the half test time. We did not compute any linear or non-linear relationship between the maximal slope value and the residual torque. Patients 1, 2, 3, 4, 6 and 8 had a higher slope value than patient 5 and a higher residual torque value. Nevertheless, patient 9 exhibited the highest residual torque and the lowest slope value. The residual torque output and the slope of decrease could be considered as the main features to characterise resistance to fatigue, therefore we computed a simple fatigue index (FI):

$$
F I=\frac{100 \cdot \mid \text { Maximum slope } \mid}{\text { Residual torque output }} .
$$

The lower its value the higher the resistance to fatigue (a null value could be encountered in case of total absence of fatigue with a zero slope). The values ranged from 2.08 (patient 9) to 27.23 (patient 6). The residual slope with a very low value in all the cases gave evidence of the plateau reached at the end of the test.

\section{PTP amplitude of CMAPs}

As is shown in Figure 3, the amplitude and general pattern of CMAPs underwent changes over the $126 \mathrm{~s}$ period of electrical stimulation. The PTP amplitude demonstrated important changes as a function of time (Figure 4, Table 2). In general, the amplitudes increased after the onset of stimulation, but soon decreased before reaching final levels below the initial amplitude, except in patient 7 (residual PTP amplitude higher by $1.4 \%$ than initial value). The time to reach the maximum PTP amplitude was longer than the time to reach the maximum torque output, and thus no relationship was encountered between torque production and PTP amplitude. The maximum relative PTP amplitude was, in general, higher than the maximum torque output and no relation was found between those maximum values. The absence of relation was also noticed between the minimum values. Therefore, the mechanical output was not a simple reflection of the PTP amplitude, ie the same PTP amplitude led to various torque output.

The minimum values were equal to the residual amplitude in three patients. In the other patients, after reaching the minimum values the PTP amplitude increased again, as was confirmed by the higher value of the residual amplitude. During this increase the 
Table 1 Torque output recorded in the patients

\begin{tabular}{|c|c|c|c|c|c|c|c|c|c|}
\hline \multirow[t]{2}{*}{ Patients } & \multicolumn{9}{|c|}{ Torque output } \\
\hline & 1 & 2 & 3 & 4 & 5 & 6 & 7 & 8 & 9 \\
\hline Max torque $(\mathrm{Nm})$ & 48.27 & 49.56 & 40.32 & 71.80 & 63.59 & 45.94 & 47.46 & 58.27 & 38.85 \\
\hline Max relative torque $(\%)$ & 113.19 & 113.56 & 100.86 & 112.87 & 106.04 & 110.56 & 103.99 & 151.19 & 104.69 \\
\hline Rising duration (s) & 14.00 & 16.00 & 2.00 & 4.00 & 2.00 & 2.00 & 2.00 & 6.00 & 4.00 \\
\hline Min relative torque $(\%)$ & 16.76 & 42.40 & 26.22 & 22.66 & 9.16 & 7.13 & 37.72 & 42.84 & 53.54 \\
\hline Total decrease $(\%)$ & 113.02 & 113.13 & 100.60 & 112.64 & 105.95 & 110.49 & 103.61 & 150.77 & 104.16 \\
\hline Decrease from initial value (\%) & 83.24 & 57.60 & 73.78 & 77.34 & 90.84 & 92.87 & 62.28 & 57.16 & 46.46 \\
\hline Decreasing duration (s) & 110.00 & 106.00 & 124.00 & 122.00 & 118.00 & 124.00 & 124.00 & 120.00 & 122.00 \\
\hline Residual torque (\%) & 17.72 & 43.85 & 26.22 & 22.66 & 9.16 & 7.13 & 37.72 & 42.84 & 53.54 \\
\hline Initial slope $(\times 100)$ & 4.91 & 3.27 & 0.43 & 4.63 & 3.02 & 5.28 & 1.99 & 23.42 & 1.96 \\
\hline Maximal slope of decrease $(\times 100)$ & -2.46 & -1.67 & -1.82 & -2.45 & -1.56 & -1.94 & -1.36 & -2.02 & -1.11 \\
\hline Time of max slope $(\mathrm{s})$ & 62.00 & 76.00 & 64.00 & 56.00 & 46.00 & 20.00 & 54.00 & 56.00 & 50.00 \\
\hline Residual slope $(\times 100)$ & -0.23 & 0.19 & -0.29 & -0.10 & -0.07 & -0.06 & -0.16 & -0.32 & -0.18 \\
\hline Fatigue index & 13.90 & 3.80 & 6.93 & 10.80 & 17.04 & 27.23 & 3.62 & 4.71 & 2.08 \\
\hline
\end{tabular}

The maximum (Max) torque is expressed both in absolute value $(\mathrm{Nm})$ and in relative value $(\%)$. The rising duration is the time to reach the maximum value of torque from the onset of torque production. The total decrease is the decrease from maximum to minimum value. The decrease duration represents the time of total decrease. The residual torque is the last torque value recorded. The calculation of the slope and the fatigue index are explained in the text

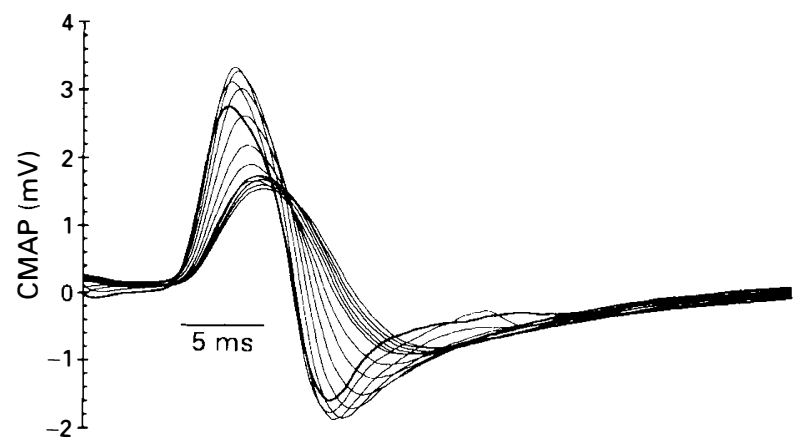

Figure 3 Typical evolution of the CMAPs along the fatigue test obtained in patient 9 . The bold line represents the initial CMAP. The time interval between each plotted CMAP is $10 \mathrm{~s}$

torque output still decreased. Considering the rate of decrease, all the curves plateaued by the end of the test as confirmed by the very low slope value.

\section{Latencies LO, duration L1 and L2}

The latency L0 (Figure 5a, Table 3), from the onset of stimulation to the onset of the CMAP, was found to be on the average $7.09 \mathrm{~ms}$ (from 6.53 to $7.67 \mathrm{~ms}$ ) from the onset of stimulation with a low dispersion $( \pm 0.13)$. This latency increased in all the patients along the test but to a limited extent (increase equal or less than $20 \%$ for the final value). The variations from the minimum values to the maximum were higher (from 13.27 to $27.83 \%$ ). The latency by the end of the $126 \mathrm{~s}$ was lower than the maximum value encountered, ie this latency plateaued or slightly decreased. L0 reflected the nerve conduction velocity and the NMJ transmission and was relatively affected by the continuous stimulation. We computed
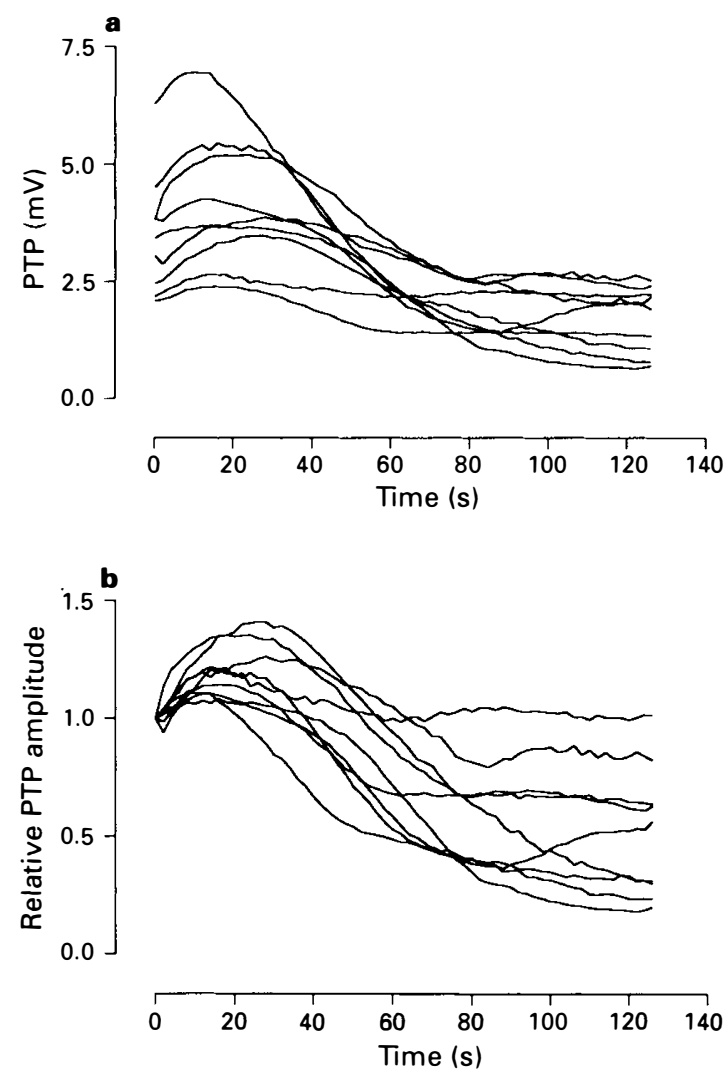

Figure 4 (a) Absolute value of PTP amplitude calculated in the nine patients and expressed in $\mathrm{mV}$; (b) values are normalised with respect to initial value

the relationship between relative $\mathrm{L} 0$ and relative torque output. In one patient we calculated a non-linear relation (one phase exponential decay) with a high value for the regression coefficient $\left(r^{2}=0.91\right)$. In the 
Table 2 Peak to peak amplitude

\begin{tabular}{|c|c|c|c|c|c|c|c|c|c|}
\hline \multirow[t]{2}{*}{ Patients } & \multicolumn{9}{|c|}{ Peak to peak amplitude } \\
\hline & 1 & 2 & 3 & 4 & 5 & 6 & 7 & 8 & 9 \\
\hline Max amplitude (mV) & 6.95 & 3.71 & 4.25 & 2.39 & 5.45 & 3.48 & 2.66 & 3.86 & 5.20 \\
\hline Max relative amplitude (\%) & 110.79 & 108.19 & 110.50 & 114.42 & 120.72 & 141.08 & 121.72 & 126.25 & 135.46 \\
\hline Rising duration (s) & 10.00 & 16.00 & 12.00 & 16.00 & 16.00 & 26.00 & 14.00 & 28.00 & 24.00 \\
\hline Total decrease $(\%)$ & 110.49 & 108.00 & 110.13 & 113.79 & 120.48 & 140.77 & 120.74 & 125.46 & 134.85 \\
\hline Min relative amplitude $(\%)$ & 30.32 & 18.21 & 36.81 & 63.73 & 23.76 & 30.98 & 98.49 & 79.34 & 60.86 \\
\hline Decrease from initial value $(\%)$ & 69.68 & 81.79 & 63.19 & 36.27 & 76.24 & 69.02 & 1.51 & 20.66 & 39.14 \\
\hline Decreasing duration (s) & 116.00 & 106.00 & 76.00 & 110.00 & 104.00 & 96.00 & 48.00 & 56.00 & 100.00 \\
\hline Residual amplitude (\%) & 30.32 & 20.33 & 56.30 & 63.73 & 23.76 & 31.67 & 101.37 & 82.28 & 62.89 \\
\hline Initial slope $(\times 100)$ & 1.63 & 1.41 & -0.69 & 0.84 & 1.84 & 1.24 & 2.02 & -3.07 & 6.75 \\
\hline Maximal slope of decrease $(\times 100)$ & -2.31 & -2.32 & -2.37 & -2.01 & -2.63 & -2.31 & -2.84 & -2.16 & -2.58 \\
\hline Time of maximal slope (s) & 34.00 & 56.00 & 48.00 & 32.00 & 44.00 & 64.00 & 22.00 & 74.00 & 46.00 \\
\hline Residual slope $(\times 100)$ & -0.08 & -0.03 & 0.12 & -0.25 & -0.25 & -0.40 & 0.15 & 0.13 & -0.40 \\
\hline
\end{tabular}

The maximum (Max) PTP amplitude is expressed both in absolute value $(\mathrm{mV})$ and in relative value (\%). The rising duration is the time to reach the maximum value of PTP amplitude from the onset of stimulation. The total decrease is the decrease from maximum to minimum value. The decrease duration represents the time of total decrease. The residual amplitude is the last value recorded. The calculation of the slope and the fatigue index are explained in the text
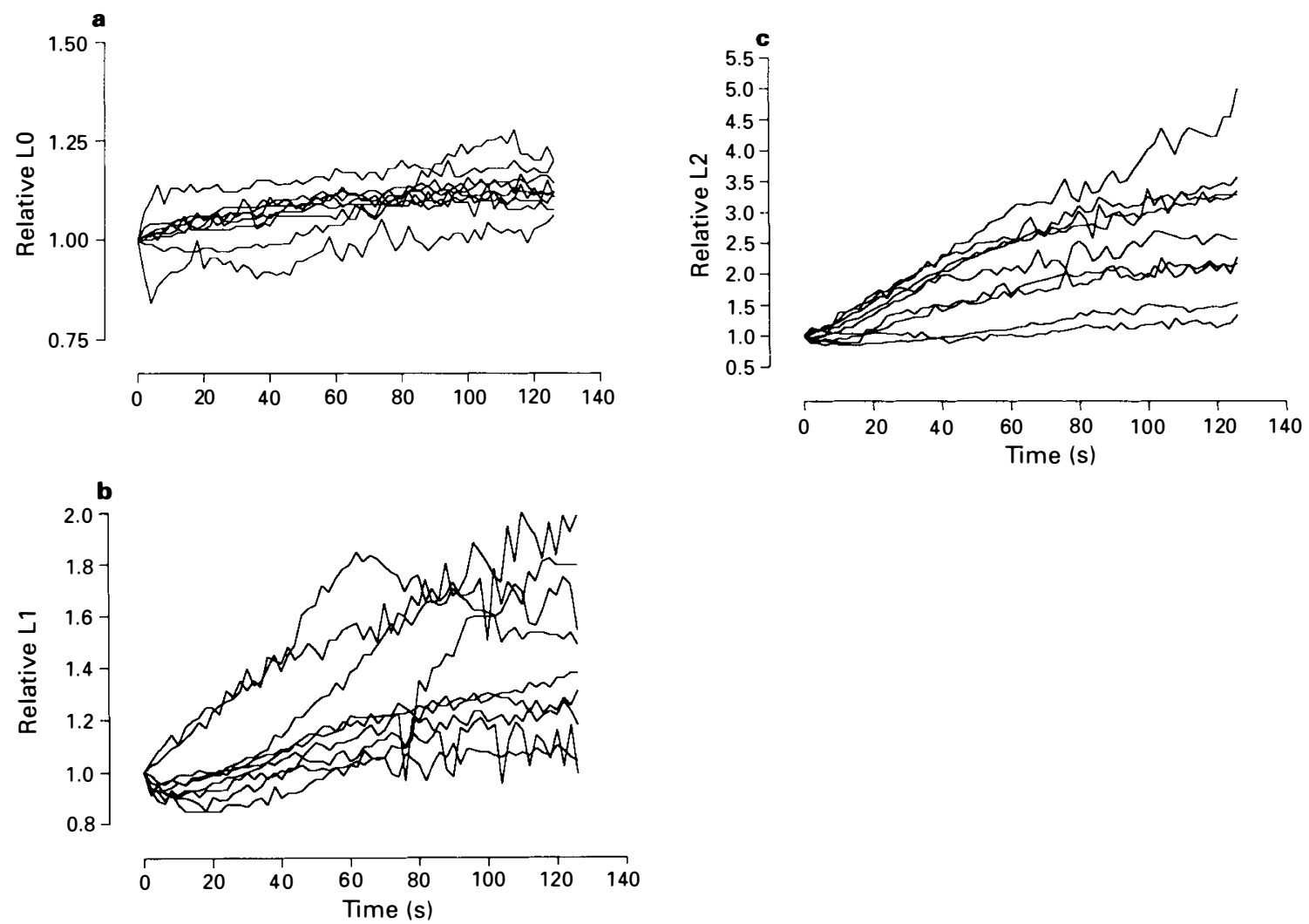

Figure 5 (a) Evolution of the relative latency L0 computed from the onset of stimulation to the onset of CMAP for the nine patients along the fatigue test; (b) evolution of the relative duration L1 computed from the onset of CMAP to the first peak; (c) evolution of the relative PTP duration L2 computed from the first peak to the second peak

other patients, a linear relation was computed ( $r^{2}$ from 0.63 to 0.92 ) with a significant deviation from zero $(P<0.0001)$.

L1 (Figure 5b, Table 4) was the duration from the onset of the CMAP to the maximum value. The variation between patients was higher than for L0 $(6.71 \pm 0.51 \mathrm{~ms}$, range $4.93-9.87 \mathrm{~ms})$. In seven patients, the initial slope was found to be negative, ie the 
Table 3 Initial latency L0

\begin{tabular}{|c|c|c|c|c|c|c|c|c|c|}
\hline \multirow[t]{2}{*}{ Patients } & \multicolumn{9}{|c|}{ Initial latency (LO) } \\
\hline & 1 & 2 & 3 & 4 & 5 & 6 & 7 & 8 & 9 \\
\hline Initial latency (ms) & 6.53 & 7.67 & 7.00 & 7.47 & 6.80 & 7.13 & 7.00 & 7.53 & 6.67 \\
\hline Max latency (ms) & 7.40 & 9.80 & 8.00 & 8.60 & 7.93 & 8.13 & 8.13 & 8.00 & 8.00 \\
\hline Time for max latency (s) & 62.00 & 114.00 & 106.00 & 124.00 & 116.00 & 116.00 & 122.00 & 126.00 & 80.00 \\
\hline Final latency (\%) & 112.24 & 120.00 & 107.62 & 110.71 & 111.76 & 112.15 & 114.29 & 106.19 & 120.00 \\
\hline Initial slope $(\times 100)$ & 3.06 & 0.87 & -0.95 & 0.00 & 0.98 & 0.00 & 0.95 & -8.85 & 7.00 \\
\hline Decreasing time (s) & 0.00 & 0.00 & 8.00 & 0.00 & 0.00 & 0.00 & 0.00 & 4.00 & 0.00 \\
\hline Total increase (\%) & 13.27 & 27.83 & 17.14 & 15.18 & 16.67 & 14.02 & 16.19 & 22.12 & 20.00 \\
\hline Increase from init (\%) & 13.27 & 27.83 & 14.29 & 15.18 & 16.67 & 14.02 & 16.19 & 6.19 & 20.00 \\
\hline
\end{tabular}

The decreasing time is calculated from the onset of stimulation to the minimum value of L0. The final latency is the last value recorded. The total increase is calculated from the minimum to the maximum value

Table $4 \mathrm{~L} 1$ is the duration from the onset of CMAP to reach the first peak

\begin{tabular}{|c|c|c|c|c|c|c|c|c|c|}
\hline \multirow[t]{2}{*}{ Patients } & \multicolumn{9}{|c|}{ L1 } \\
\hline & 1 & 2 & 3 & 4 & 5 & 6 & 7 & 8 & 9 \\
\hline Initial duration (ms) & 6.87 & 7.40 & 7.00 & 6.87 & 5.20 & 5.13 & 7.13 & 9.87 & 4.93 \\
\hline Max duration (ms) & 9.00 & 12.93 & 11.93 & 9.47 & 9.60 & 10.27 & 8.73 & 10.93 & 6.27 \\
\hline Time for max duration (s) & 126.00 & 122.00 & 90.00 & 124.00 & 62.00 & 110.00 & 74.00 & 90.00 & 122.00 \\
\hline Final duration (\%) & 131.07 & 154.05 & 148.57 & 137.86 & 179.49 & 198.70 & 99.07 & 104.05 & 117.57 \\
\hline Initial slope $(\times 100)$ & -5.83 & -7.21 & -3.81 & -5.83 & 2.56 & 3.90 & -0.93 & -8.78 & -6.76 \\
\hline Decreasing time (s) & 4.00 & 12.00 & 12.00 & 8.00 & 0.00 & 0.00 & 6.00 & 18.00 & 6.00 \\
\hline Total increase $(\%)$ & 37.86 & 90.09 & 75.24 & 46.60 & 84.62 & 100.00 & 27.10 & 25.68 & 39.19 \\
\hline Increase from init (\%) & 31.07 & 74.77 & 70.48 & 37.86 & 84.62 & 100.00 & 22.43 & 10.81 & 27.03 \\
\hline
\end{tabular}

duration decreased during the first seconds (from 4 to $18 \mathrm{~s})$. Then it increased to reach values ranging from 104 to $199 \%$ of initial values. This duration was, therefore, more affected by the test than L0. It was not possible to compute a relation between relative $\mathrm{L} 1$ and relative torque output in patient 7 . In the other patients, we calculated a non-linear relation (one phase exponential decay) in one patient $\left(r^{2}=0.97\right)$ and a linear relation for the other patients (from 0.87 to 0.97 for $r^{2}$ ) and a significant deviation from zero $(P<0.0001)$.

The peak to peak duration L2 (Figure 5c, Table 5) reflected the conduction velocity along the muscle fibres, from the maximum to the minimum of the CMAPs. Its initial value ranged from 3.87 to $10.47 \mathrm{~ms}$. Therefore, the dispersion around the mean value $(6.72 \mathrm{~ms})$ was quite high $( \pm 0.88 \mathrm{~ms})$. In five patients the initial slope was found to be negative. In patient 8 , the second value (recorded at $t=2 \mathrm{~s}$ ) increased, explaining the positive slope, and then decreased to reach a minimum at $42 \mathrm{~s}$. The final duration (from 134 to $500 \%$ of initial value) revealed a clear reduction in the conduction velocity. L2 was extremely affected by the test except in patients 8 and 9 who had the best

Table 5 Peak to peak duration L2 computed from the maximum to the minimum of CMAPs

\begin{tabular}{|c|c|c|c|c|c|c|c|c|c|}
\hline \multirow[t]{2}{*}{ Patients } & \multicolumn{9}{|c|}{$L 2$} \\
\hline & 1 & 2 & 3 & 4 & 5 & 6 & 7 & 8 & 9 \\
\hline Initial duration (ms) & 5.00 & 4.33 & 4.07 & 3.87 & 8.60 & 10.33 & 6.00 & 10.47 & 7.80 \\
\hline Max duration (ms) & 16.73 & 21.67 & 14.53 & 8.53 & 29.07 & 27.80 & 13.67 & 14.07 & 12.00 \\
\hline Time for max duration (s) & 126.00 & 126.00 & 126.00 & 118.00 & 100.00 & 102.00 & 126.00 & 126.00 & 126.00 \\
\hline Final duration (\%) & 334.67 & 500.00 & 357.38 & 217.24 & 327.91 & 255.48 & 227.78 & 134.39 & 153.85 \\
\hline Initial slope $(\times 100)$ & 2.67 & 9.23 & -3.28 & -5.17 & 6.20 & -2.58 & -8.89 & 13.38 & -11.97 \\
\hline Decreasing time (s) & 0.00 & 0.00 & 2.00 & 6.00 & 0.00 & 2.00 & 4.00 & 42.00 & 14.00 \\
\hline Total increase (\%) & 234.67 & 400.00 & 260.66 & 129.31 & 237.98 & 171.61 & 137.78 & 45.86 & 68.38 \\
\hline Increase from init (\%) & 234.67 & 400.00 & 257.38 & 120.69 & 237.98 & 169.03 & 127.78 & 34.39 & 53.85 \\
\hline
\end{tabular}


performance in terms of residual torque output. Here again, for patient 7 we did not compute any relation between relative $L 2$ and relative torque output. In the other patients, we calculated a linear relation (from 0.88 to 0.97 for $r^{2}$ ) with a significant deviation from zero $(P<0.0001)$. L2 final value appeared to be significantly higher than L0 and L1 final values $(P<0.006)$. The increase from the onset of stimulation was also higher for L2 compared to L0 $(P=0.0004)$ and L1 $(P=0.006)$, and for L1 compared to L0 $(P=0.008)$. The torque output appeared to have no relation with the different latencies computed.

\section{Rectified CMAPs surface}

As is seen in the results (Figure 6, Table 6) $S$ varied greatly among patients (maximum values from 422.39 to $1813.89 \mathrm{mV} \mathrm{ms}^{-1}$ ). Considering the very high sampling frequency, the $S$ values could be considered as accurate approximations of the real surface. The $S$ increased in all of the patients from the onset of stimulation. In four patients we noticed an initial brief decrease. After reaching a maximum value (from 121.32 to $181.4 \%$ of initial values) $S$ decreased to reach a minimum value. Nevertheless, in three patients the residual surface was higher than the initial value. By the end of the test, all the surface curves plateaued as was confirmed by the low slope value. Patients 2,5 and 6 demonstrated a comparable residual surface value but
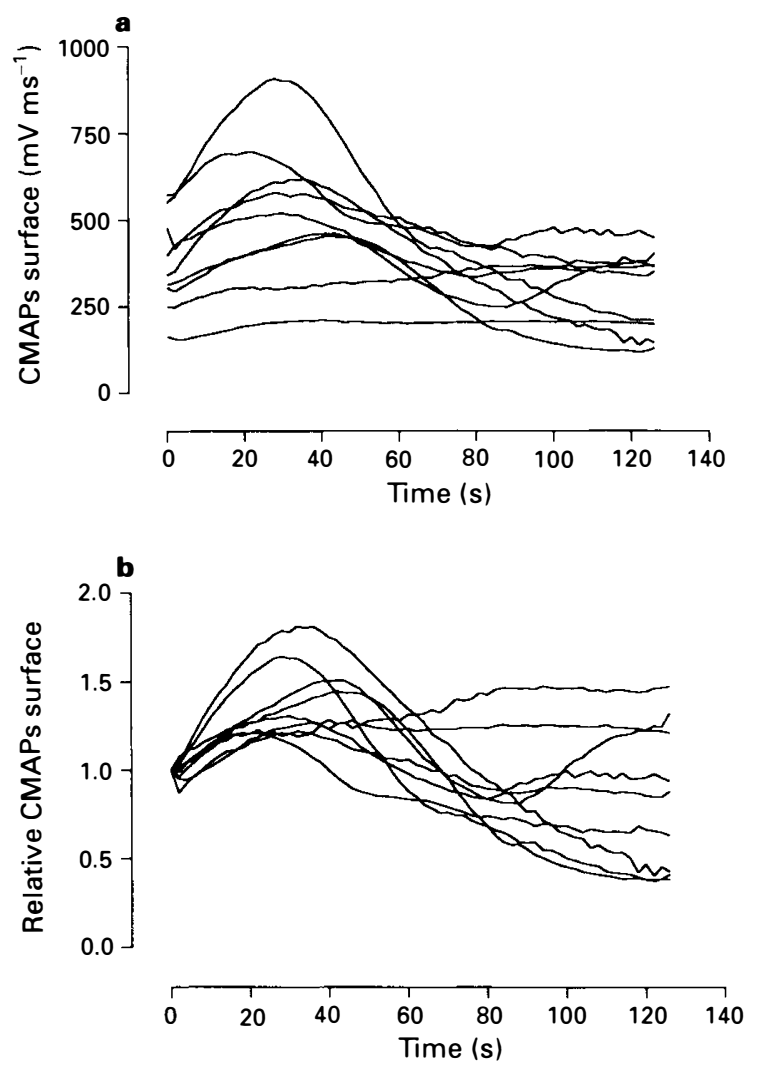

Figure 6 (a) Evolution of the absolute rectified surface of CMAPs $\left(\mathrm{mV} \mathrm{ms}^{-1}\right)$ for the nine patients along the fatigue test; (b) values are normalised with respect to initial value a different residual torque. Patients 2 and 8 had a comparable residual torque output but a different residual surface. None of the parameters computed in table were related to the torque output parameters. We did not find any relation, linear or non-linear, between the surface of the CMAPs and the torque generated.

\section{Discussion}

Although significant differences were encountered among patients with respect to mechanical and electrophysiological measures, the general pattern of responses to electrical stimulation was similar.

The resistance to fatigue, in the present study, appeared to be extremely variable among the patients. Neither the thoracic level of injury, nor the length of time after injury accounted for this variability. The rapid decrease in torque output recorded in this population of patients in other studies has shown that it is not the result of ischaemia. ${ }^{3}$

We recorded important changes in the computed parameters of the recorded CMAPs during these tests; first, we observed a reduction in amplitude and second the increase of latencies. The recorded CMAPs represent the summation of the contributing MUAP recruited with electrical stimulation. Therefore the reduction of the amplitude could result from the reduction in single-fibre events, ie a reduction in the number of contributing MUAPs or a reduction in the amplitude of the MUAPs. The increase in the computed latencies could result from the decrease in the conduction velocity or could reflect that the remaining slow fibres are still activated. Nevertheless, the nerve conduction velocity and the NMJ transmission appeared to be less affected than the muscle conduction velocity (PTP duration). The NMJ transmission could have been affected by the test as indicated by the increase of L0 but it is difficult to quantify these changes. The variation in torque output along the fatigue test appeared to be linearly related to the conduction velocity.

As is seen in the results, the amplitude of the CMAP first increased and then decreased which is in accordance with several studies. ${ }^{4,5}$ Intense stimulation of isolated mouse muscles have been reported to decrease the intracellular potassium concentration, to double the interstitial potassium concentration and to decrease the intracellular $\mathrm{pH}^{8,11,12}$ Similar changes have been reported to take place in man. The increased concentration of potassium on the extracellular side of the membrane is associated with a considerable broadening of the action potential, and results in an increased number of inexcitable cells ${ }^{13}$ which could account for the decrease in conduction velocity and reduction in amplitude. The effects of $\mathrm{pH}$, on the other hand, mediated via intracellular sites, are associated with only minor changes in action potential shape, and do not result in inexcitability in the physiological range of pH. ${ }^{11}$

The muscle deprived from voluntary control is subject to changes in its fibre typing characterised by an 
Table 6 The rectified total surface is the surface under the CMAP curve from the onset to the end of the CMAP. The residual surface is the last value recorded

\begin{tabular}{|c|c|c|c|c|c|c|c|c|c|}
\hline \multirow[t]{2}{*}{ Patients } & \multicolumn{9}{|c|}{ Total surface } \\
\hline & 1 & 2 & 3 & 4 & 5 & 6 & 7 & 8 & 9 \\
\hline Max surface $\left(\mathrm{mVms}^{-1}\right)$ & 1394.46 & 908.63 & 920.77 & 422.39 & 1813.89 & 1236.78 & 739.00 & 1160.28 & 1042.49 \\
\hline Max relative surface & 121.32 & 144.94 & 150.85 & 128.78 & 164.53 & 181.40 & 147.92 & 121.32 & 131.13 \\
\hline Initial slope $(\times 100)$ & 0.20 & 1.00 & -1.84 & -2.43 & 1.71 & 1.65 & -0.40 & -6.35 & 3.66 \\
\hline Initial decreasing time (s) & 0.00 & 0.00 & 2.00 & 4.00 & 0.00 & 0.00 & 2.00 & 2.00 & 0.00 \\
\hline Time for $\max (\mathrm{s})$ & 22.00 & 42.00 & 40.00 & 36.00 & 28.00 & 32.00 & 92.00 & 26.00 & 30.00 \\
\hline Min relative surface & 63.34 & 37.70 & 81.21 & 94.82 & 38.17 & 40.45 & 99.19 & 87.30 & 83.89 \\
\hline Time for min (s) & 126.00 & 122.00 & 88.00 & 4.00 & 120.00 & 122.00 & 2.00 & 2.00 & 80.00 \\
\hline Total decrease (\%) & 120.69 & 113.13 & 100.60 & 112.64 & 105.95 & 110.49 & 103.61 & 150.77 & 104.16 \\
\hline Residual slope $(\times 100)$ & 0.02 & -0.03 & 0.52 & -0.22 & -0.38 & -0.61 & 0.27 & 0.08 & -0.31 \\
\hline Residual surface (\%) & 63.34 & 41.16 & 132.51 & 121.35 & 38.17 & 42.56 & 147.62 & 94.00 & 88.40 \\
\hline
\end{tabular}

increase in type IIb and a decrease in type I fibres. ${ }^{14}$ Fatiguable units usually display marked reduction in amplitude and area, whereas fatigue-resistant motor units exhibit little or no change in the CMAP. ${ }^{4}$ Therefore, type IIb fibres were presumably dominant in the muscles studied. In the present study, after an initial increase and a decrease, the amplitude reaches a minimum level, which could represent the output coming from fatigue resistant fibres still activated. In general, at the end of $126 \mathrm{~s}$ the CMAP measures were found to reach relatively stable levels.

Hainaut et $a l^{5}$ said that electrical changes do not closely correlate with mechanical failure. The present results, partially agree with such a finding since we did not compute any relation between torque output and PTP amplitude and between torque output and CMAPs surface. In a previous study ${ }^{3}$ we assumed the different parts of the fatigue curve to represent a cutting out of the different metabolic phases involved in force production. The last plateau of the curve represented the output from fatigue resistant fibres (aerobic metabolism). The first part and the main slope which appeared after 10 to $20 \mathrm{~s}$, represented the anaerobic lactate metabolism where the $\mathrm{pH}$ actually decreased. This was confirmed by ${ }^{31} \mathrm{P}$ NMR studies. ${ }^{15}$

This $\mathrm{pH}$ decrease, resulting from lactate production when ATP demands exceeds maximal aerobic capacity, has been reported ${ }^{1,5,13}$ to inhibit the interaction between the contractile proteins. In fact, increase concentration of $\mathrm{H}^{+}$can decrease the capacity to generate ATP through an inhibitory effect on glycolysis and through a decrease in the $\mathrm{PCr}$ level. However, if the accumulation of $\mathrm{H}^{+}$appears to be related to fatigue it is not the main cause, ${ }^{1}$ and according to Salhin et al ${ }^{12}$ the capacity to generate force in vivo is not limited by a high intracellular concentration of $\mathrm{H}^{+}$(the time course for restoration of force and normal $\left[\mathrm{H}^{+}\right]$is different). Nevertheless, the same authors reported the fact that acidosis does not impair contractile force but rather appears to limit endurance during a sustained contraction which was the case in the present study. The slowing of the conduction was identified by the broadening of the action potential.

Fatigue can be considered as a limit of functional electrical stimulation use in spinal cord injured patients. Nevertheless, none of the patients were previously trained with electrical stimulation. Therefore, it will be interesting to monitor the changes in CMAPs and torque production in regularly trained patients. Training has been reported to modify fibre typing within the stimulated muscle and this protocol can be applied to quantify those changes.

\section{References}

1 Edwards RHT, Gibson H. Perspectives in the study of normal and pathological skeletal muscle. In: Atlan G, Beliveau L, Bouissou P, (eds). Muscle Fatigue. Biochemical and Physiological Aspects. Masson: Paris, 1991, pp 3-15.

2 Merletti R, Lo Conte LR, Orizio C. Indices of muscle fatigue. J Electromyogr Kinesiol 1: 20-33.

3 Rabischong E, Guiraud D. Determination of fatigue in the electrically stimulated quadriceps muscle and relative effect of ischemia. J Biomed Eng 1993; 15: 443-450.

4 Enoka RM et al. Fatigue-related changes in motor unit action potentials of adult cats. Muscle Nerve 1992; 14: 138-150.

5 Hainaut K, Duchateau J. Muscle fatigue, effects of training and disuse. Muscle Nerve 1989; 12: 660-669.

6 Duchateau J, Hainaut K. Electrical and mechanical changes in immobilised human muscle. $J$ Appl Physiol 1987; 62: 2168-2173.

7 Robinson GA, Enoka RM, Stuart DG. Immobilization-induced changes in motor unit force and fatigability in the cat. Muscle Nerve 1991; 14: 563-573.

8 Moussavi RS et al. Nonmetabolic fatigue in exercising human muscle. Neurology 1989; 39: 1222-1226.

9 Levy M, Mizrahi J, Susak Z. Recruitment, force and fatigue characteristics of quadriceps muscles of paraplegics isometrically activated by surface functional electrical stimulation. J Biomed Eng 1990; 12: 150-156.

10 Rabischong E, Ohanna F. Effects of FES on evoked muscular output in paraplegics. Paraplegia 1992; 30: 467-473.

11 Juel C. Muscle action potential propagation velocity changes during activity, Muscle Nerve 1988; 11: 714-719.

12 Sahlin K, Ren JM. Relationship of contraction capacity to metabolic changes during recovery from a fatiguing contraction. J Appl Physiol 1989; 67: 648-654.

13 Holloszy JO. Muscle metabolism during exercise. Arch Phys Med Rehabil 1982; 63: 231-233.

14 Salmons S, Henriksson J. The adaptative response of skeletal muscle to increased use. Muscle Nerve 1981; 4: 94-105.

15 Levy M, Kushnir T, Mizrahi J, Itzchak Y. In vivo 31P NMR studies of paraplegics' muscles activated by functional electrical stimulation. Magn Reson Med 1993; 29: 53-58. 